\title{
Characterization of circulating transfer RNA-derived RNA fragments in cattle
}

\author{
Eduardo Casas*, Guohong Cai and John D. Neill \\ National Animal Disease Center, United States Department of Agriculture - Agricultural Research Service, Ames, IA, USA
}

The objective was to characterize naturally occurring circulating transfer RNA-derived RNA fragments (tRFs) in cattle ${ }^{1}$. Serum from eight clinically normal adult dairy cows was collected, and small non-coding RNAs were extracted immediately after collection and sequenced by Illumina MiSeq. Sequences aligned to transfer RNA (tRNA) genes or their flanking sequences were characterized. Sequences aligned to the beginning of $5^{\prime}$ end of the mature tRNA were classified as tRF5; those aligned to the $3^{\prime}$ end of mature

OPEN ACCESS

Edited by:

Graziano Pesole,

University of Bari, Italy

Reviewed by:

Matteo Chiara,

Università degli studi di Milano, Italy

Mainá Bitar

Universidade Federal de Minas

Gerais, Brazil

*Correspondence:

Eduardo Casas,

National Animal Disease Center,

United States Department of

Agriculture - Agricultural Research

Service,

1920 Dayton Avenue, Ames,

IA 50010, USA

eduardo.casas@ars.usda.gov

Specialty section:

This article was submitted to

RNA,

a section of the journal

Frontiers in Genetics

Received: 26 May 2015 Accepted: 07 August 2015

Published: 24 August 2015

Citation:

Casas E, Cai G and Neill JD (2015) Characterization of circulating transfer RNA-derived RNA fragments in cattle. Front. Genet. 6:271. doi: 10.3389/fgene.2015.00271 tRNA were classified as tRF3; and those aligned to the beginning of the $3^{\prime}$ end flanking sequences were classified as tRF1. There were 3,190,962 sequences that mapped to transfer RNA and small non-coding RNAs in the bovine genome. Of these, 2,323,520 were identified as tRF5s, 562 were tRF3s, and 81 were tRF1s. There were 866,799 sequences identified as other small non-coding RNAs (microRNA, rRNA, snoRNA, etc.) and were excluded from the study. The tRF5s ranged from 28 to 40 nucleotides; and $98.7 \%$ ranged from 30 to 34 nucleotides in length. The tRFs with the greatest number of sequences were derived from tRNA of histidine, glutamic acid, lysine, glycine, and valine. There was no association between number of codons for each amino acid and number of tRFs in the samples. The reason for tRF5s being the most abundant can only be explained if these sequences are associated with function within the animal.

Keywords: cattle, RNA-seq, small non-coding RNA, tRF, transfer RNA fragments

\section{Introduction}

High throughput sequencing has allowed the identification of small non-coding RNA (Cole et al., 2009; Lee et al., 2009). Transfer RNA-derived RNA fragments (tRFs) are a component of small non-coding RNA (Haussecker et al., 2010). MicroRNAs (miRNA), small interfering RNA (siRNA), and Piwi-interfering RNA (piRNA), are also considered sncRNA. MicroRNAs have been the most studied because of their regulatory functions (Bartel, 2009; Stewart et al., 2013).

The tRFs were previously considered a degradation product; however, they were associated with regulation of translation in the cell (Cole et al., 2009; Garcia-Silva et al., 2012; Sobala and Hutvagner, 2013). It has been established tRFs are not randomly generated and have distinct size (Kumar et al., 2014). Their nomenclature is based on the processing site of the transfer RNA

\footnotetext{
${ }^{1}$ Mention of trade name, proprietary product, or specified equipment does not constitute a guarantee or warranty by the USDA and does not imply approval to the exclusion of other products that may be suitable. USDA is an Equal Opportunity Employer.
} 
(tRNA): tRFs processed from the $5^{\prime}$ or $3^{\prime}$ end of the mature RNA are termed tRF5 and tRF3, respectively, while those produced from the $3^{\prime}$ sequence cleaved from the immature tRNA are designated tRF1 (Lee et al., 2009; Haussecker et al., 2010; Kumar et al., 2014). These RNAs are the second most abundant, after microRNAs (Lee et al., 2009).

It has been suggested that tRFs have similar function as microRNAs (Haussecker et al., 2010; Garcia-Silva et al., 2012). MicroRNA and tRFs are created by analogous processes in the cell, where the last step is its cleavage by the endonuclease Dicer (Garcia-Silva et al., 2012; Sobala and Hutvagner, 2013). Once cleaved by Dicer, tRFs are involved in RNA silencing through their association with Argonaut proteins.

It has been proposed that tRFs are produced in the bone marrow and in the immune cells (Nolte-'t Hoen et al., 2012), although other cells may also have the ability to produce them (Thompson and Parker, 2009; Ivanov et al., 2011). These molecules have been identified as being involved in inhibition of gene expression, in cell stress (Thompson and Parker, 2009), and in virus replication (Wang et al., 2013). Given that $t R F$ s play a role in inhibiting gene expression or in virus replication, it is possible these functions may be involved in mediating infection-induced defense response by organisms (Zhang et al., 2014).

It is now known that tRFs are highly abundant molecules in different cell lines and tissues from human and mouse (Bartel, 2009; Kumar et al., 2014), and have also been identified in databases from plants (Loss-Morais et al., 2013). A single study has characterized tRFs associated with human cell lines challenged with respiratory syncytial virus (Wang et al., 2013), but no attempt has been made to characterize naturally occurring tRFs in homeostatic cattle, which is the objective of this study.

\section{Materials and Methods}

\section{Animals}

Serum from eight adult dairy cows was obtained from the dairy barn from the National Animal Disease Center, in Ames, IA, USA. Bleeding of animals was done according to the management protocol approved by the Animal Care and Use Committee of the Institution. Fresh serum was obtained from eight adult female Holstein cows by jugular venipuncture in SST vacutainer tubes (BD, Franklin Lakes, NJ, USA). The vacutainer tubes were incubated at $37^{\circ} \mathrm{C}$ degrees for $30 \mathrm{~min}$ and were centrifuged at $1250 \times \mathrm{g}$ for $30 \mathrm{~min}$. The isolated serum samples were placed into $2 \mathrm{ml}$ vials and were stored at $-80^{\circ} \mathrm{C}$ until processed.

\section{tRF Isolation}

The tRFs were isolated from the serum samples using the miRNeasy Serum/Plasma kit (QIAGEN, Germantown, MD, USA) using $200 \mathrm{ul}$ of serum sample. The tRFs was extracted according to manufactures direction and the samples were eluted in $14 \mathrm{ul}$ of RNase free water. After extraction $1 \mathrm{ul}$ of each sample were run using the Small RNA chip on an Agilent 2100 Bioanalyzer (Agilent Technologies, Santa Clara, CA, USA) to quantify the serum extracted from the samples. The tRFs concentration was determined by using a 10-40 nucleotide gate.

\section{Library Preparation}

Each extracted serum sample was used to prepare sequencing libraries. The libraries were prepared using the NEBNext Multiplex Small RNA Library Prep Set for Illumina Set 1 and 2 (New England BioLabs, Ipswich, MA, USA). The libraries were individually index with the Illumina 1-24 indexed primers. Six microliters (6 ul) of each animal's isolated serum was used in library preparation according to manufacturer's instructions. After the library preparation the libraries were cleaned up and concentrated using the QIAquick PCR purification kit (QIAGEN, Germantown, MD, USA) from 100 to $27.5 \mathrm{ul}$. The quality and quantity of the libraries were determined by running $1 \mathrm{ul}$ of each library on a DNA 1000 chip on an Agilent 2100 Bioanalyzer (Agilent Technologies, Santa Clara, CA, USA). The concentration of each indexed library was determined by using a 135-170 nucleotide gate. All the indexed libraries were then pooled and size selected. Five nanograms of each indexed library used to make the pool. The total volume of the pool was 246.5 ul. The pool was concentrated using the QIAquick PCR purification kit (QIAGEN, Germantown, $\mathrm{MD}$, USA) to $35 \mathrm{ul}$ of RNase free water. The pool was then size selected using the Pippin Prep on a 3\% Agarose gel without added Ethidium Bromide (SAGE Sciences, Beverly, MA, USA) with a size selection of 142-170 nt according to manufactures instructions. After the gel was run the pools were concentrated using the QIAquick PCR purification kit (QIAGEN, Germantown, MD, USA) by eluting in $32 \mathrm{ul}$ of RNase free water. One microliter of the size selected library pool was run using a High sensitivity DNA chip Agilent 2100 Bioanalyzer (Agilent Technologies, Santa Clara, CA, USA). The concentration was determined by using a 135-170 nucleotide gate. The final concentration of the size selected pool library was $1.5 \mathrm{nM}$ and the pool was stored at $-20^{\circ} \mathrm{C}$.

\section{Sequencing the Library Pool}

The pooled size selected library was sequenced on a MiSeq using the MiSeq Sequencing Kit v2 50 Cycles (Illumina, San Diego, CA, USA) in the Sequencing Core Facility at the National Animal Disease Center (NADC).

\section{Data Analysis}

The quality of Illumina sequences was inspected using FastQC v0.11.2 $2^{2}$ The Illumina adapter was removed using fastx_clipper program in the fastx toolkit ${ }^{3}$. Multiple occurrences of unique reads were merged using a custom script. Reads 18-40 bp were used in downstream analysis. They were first mapped to Bos taurus genome (ENSEMBL UMD3.1.75) using Novoalign software (Novocraft Technologies) allowing two mismatches (-o SAM -o FullNW -g 99 -r Random -t 60). Those aligned to the genome were then mapped to a database containing different annotated genome features to determine their origin (-o SAM -o FullNW -g 99 -r All -t 60): genomic tRNA sequences were downloaded from http://gtrnadb.ucsc.

\footnotetext{
${ }^{2}$ http://www.bioinformatics.babraham.ac.uk/projects/fastqc/

${ }^{3}$ http://hannonlab.cshl.edu/fastxtoolkit/
} 
edu/; mitochondrial tRNA, cDNA, and other non-coding RNA sequences were downloaded from ENSEMBL version 75. To account for tRFs derived from tRNA precursors, such as tRF1, 40-bp flanking sequences on both ends of tRNA genes were retrieved from the genome and appended to the tRNA genes. The Illumina reads aligned to tRNA genes or their flanking sequences were further characterized. They were aligned to Bos taurus tRNA database using BLASTN and the results were processed using a custom script. Those perfectly aligned to the beginning of mature tRNAs were classified as tRF5; those perfectly aligned to the end of mature tRNA, with or without the CCA sequences at the $3^{\prime}$ end, were classified as tRF3; and those aligned to the beginning of the $3^{\prime}$ end flanking sequences without mismatch were classified as tRF1. After tRF5, tRF3, and tRF1 sequences have been determined, their occurrences in Illumina sequences from individual animals were obtained using a custom script. Sequences have been submitted to NCBI Short Read Archive ${ }^{4}$, under BioProject accession PRJNA289352.

All sequences were obtained from sera, and similar tRF composition and count of sequences were expected for all samples. Sequences were normalized using the following methodology:

Normalized Counts $=$ Raw Counts $*$ mean library size/library size of individual sample.

${ }^{4}$ http://www.ncbi.nlm.nih.gov/genbank/
Statistical correlations were determined between normalized tRF5s and number of codons per amino acid, and between normalized tRF5s and number of genes, using the PROC CORR procedure from SAS (SAS Inst. Inc., Cary, NC, USA).

\section{Results}

There were a total of 5,078,907 sequences obtained in the present study. From these, 4,466,139 sequences were between 18 and 40 nucleotides in length. There were 3,190,962 sequences that mapped to tRNA and small non-coding RNAs in the bovine genome. Of these, 2,323,520 were identified as tRF5s, 562 were tRF3s, and 81 were tRF1s. There were 866,799 sequences identified as small non-coding RNAs (microRNA, rRNA, snoRNA, etc.) and were excluded from the study.

For identification purposes, the nomenclature of tRFs will be according to the tRNA of origin. The number of tRF5 sequences by size is shown in Table 1. The tRF5s ranged from 28 to 40 nucleotides. There were fewer than 2,000 sequences for each tRF5 ranging from 15 to 18 nucleotides in length, and fewer than 4,000 sequences for each tRF5 ranging from 19 to 27 nucleotides in length. Each sequence ranging from 15 to 27 nucleotides in length represented less than $0.2 \%$ of all the sequences and were removed from the study. Sequences ranging from 30 to 34 nucleotides in length comprised $98.7 \%$ of all sequences. The largest number of sequences was observed at 31 nucleotides (27.6\%), followed by 34 nucleotides $(25.9 \%)$. These two nucleotide sizes account

TABLE 1 | Number of transfer RNA derived RNA fragments (tRF5) according to length in nucleotides (nt).

\begin{tabular}{|c|c|c|c|c|c|c|c|c|c|c|c|c|c|c|}
\hline \multirow[b]{2}{*}{ tRF5 } & \multicolumn{13}{|c|}{ Length (nt) } & \multirow[b]{2}{*}{ Total } \\
\hline & 28 & 29 & 30 & 31 & 32 & 33 & 34 & 35 & 36 & 37 & 38 & 39 & 40 & \\
\hline tRF5-Ala & 32 & 584 & 2304 & 387 & 172 & 1 & 0 & 0 & 0 & 0 & 0 & 0 & 0 & 3480 \\
\hline tRF5-Arg & 0 & 0 & 0 & 1 & 0 & 3 & 0 & 0 & 0 & 0 & 0 & 0 & 0 & 4 \\
\hline tRF5-Asn & 0 & 0 & 1 & 0 & 0 & 0 & 0 & 0 & 0 & 0 & 0 & 0 & 0 & 1 \\
\hline tRF5-Asp & 0 & 0 & 0 & 0 & 0 & 5 & 46 & 8 & 3 & 0 & 0 & 0 & 0 & 62 \\
\hline tRF5-Cys & 4 & 3 & 5 & 45 & 169 & 54 & 3 & 2 & 0 & 0 & 0 & 0 & 0 & 285 \\
\hline tRF5-Gln & 27 & 29 & 1381 & 1044 & 10 & 79 & 105 & 11 & 7 & 0 & 0 & 0 & 5 & 2698 \\
\hline tRF5-Glu & 761 & 5235 & 45495 & 356687 & 112717 & 134146 & 22158 & 5165 & 916 & 41 & 58 & 1291 & 1047 & 685717 \\
\hline tRF5-Gly & 2811 & 7191 & 311707 & 64428 & 34303 & 969 & 203 & 144 & 545 & 3 & 6 & 1 & 71 & 422382 \\
\hline tRF5-His & 38 & 454 & 72659 & 119840 & 76384 & 8686 & 553427 & 27 & 10 & 0 & 0 & 0 & 2 & 831527 \\
\hline tRF5-Ile & 0 & 0 & 0 & 0 & 0 & 0 & 0 & 0 & 0 & 0 & 0 & 0 & 0 & 0 \\
\hline tRF5-Leu & 0 & 0 & 0 & 0 & 1 & 3 & 13 & 0 & 0 & 0 & 0 & 0 & 0 & 17 \\
\hline tRF5-Lys & 454 & 37 & 1158 & 16529 & 26139 & 3400 & 895 & 50 & 29 & 2 & 1 & 6 & 69 & 48769 \\
\hline tRF5-Met & 0 & 0 & 8 & 56 & 0 & 0 & 0 & 0 & 0 & 0 & 0 & 0 & 0 & 64 \\
\hline tRF5-Phe & 0 & 0 & 0 & 0 & 0 & 0 & 1 & 0 & 0 & 0 & 0 & 0 & 0 & 1 \\
\hline tRF5-Pro & 286 & 301 & 1250 & 2295 & 1001 & 1047 & 4 & 2 & 0 & 0 & 0 & 0 & 0 & 6189 \\
\hline tRF5-Ser & 0 & 0 & 0 & 0 & 2 & 0 & 0 & 0 & 0 & 0 & 0 & 0 & 0 & 2 \\
\hline tRF5-Stop & 0 & 1 & 0 & 0 & 0 & 0 & 7 & 391 & 26 & 13 & 0 & 0 & 0 & 438 \\
\hline tRF5-Thr & 0 & 1 & 19 & 49 & 44 & 0 & 0 & 0 & 0 & 0 & 0 & 0 & 0 & 113 \\
\hline tRF5-Trp & 0 & 0 & 0 & 0 & 0 & 0 & 0 & 0 & 0 & 0 & 0 & 0 & 0 & 0 \\
\hline tRF5-Tyr & 0 & 0 & 0 & 0 & 0 & 0 & 0 & 0 & 0 & 0 & 0 & 0 & 0 & 0 \\
\hline tRF5-Val & 59 & 888 & 1321 & 80220 & 160655 & 54321 & 24171 & 30 & 53 & 40 & 3 & 8 & 5 & 321774 \\
\hline Total & 4472 & 14724 & 437308 & 641581 & 411597 & 202714 & 601033 & 5830 & 1589 & 99 & 68 & 1306 & 1199 & 2323520 \\
\hline Proportion & 0.002 & 0.006 & 0.188 & 0.276 & 0.177 & 0.087 & 0.259 & 0.002 & 0.001 & 0.00 & 0.00 & 0.001 & 0.001 & \\
\hline
\end{tabular}


for $53.5 \%$ of the total number of sequences. The most abundant tRF5s were tRF5-His, tRF-Glu, tRF5-Lys, tRF5-Gly, and tRF5Val. For tRF5-His, the majority were 34 nucleotides in length, followed by a population of 31 nucleotides. For tRF5-Glu, the largest number was 31 nucleotides, followed by the second most abundant of 33 nucleotides. For tRF5-Gly, the most common was 30 nucleotides in length, followed by 31 nucleotides. For tRF5Lys and tRF5-Val, the most common was 32 nucleotides in length, followed by 31 nucleotides in length. Regardless of the tRF5, in all cases, the length with the most number of sequences had more than double the amount when compared to the second most common.

There are several patterns regarding length of tRF5 (Table 1). tRF5-His had the largest number of sequences and most ranged from 28 to 36 nucleotides in length. The tRF5 for tRF5-Glu, tRF5Gly, tRF5-Lys, and tRF5-Val, ranged from 28 to 40 nucleotides. Although tRF5-Ala had more than 1,000 sequences, the length ranged from 28 to 33 nucleotides in length. The length of tRF5Gln ranged from 28 to 36 nucleotides.

The number of tRF5s for each animal in the study is shown on Table 2. There were more than 2.3 million tRF5s, from approximately 5.1 million total sequences (46\% of the total sequences). The number of tRF5s for each animal ranged from 259,480 to 316,580 , with an average of 290,440 . The number of tRF5s identified for each animal is consistent across all animals, without any given animal having extreme numbers of sequences.

In Table 2 it was observed there were five populations of tRF5 with more than 1000 per animal (tRF5-Glu, tRF5-Gly, tRF5-His,
tRF5-Lys, and tRF5-Val). tRF5-His had the largest number per animal, followed by tRF5-Glu, tRF5-Gly, tRF5-Val, and tRF5-Lys. Three tRF5s, tRF5-Ala, tRF5-Gln, and tRF5-Pro, had more than 100 and fewer than 1000 sequences per animal. Two tRF5s, tRF5Trp and tRF5-Tyr, had no sequences. The remaining tRF5s had at least 1 , but fewer than 100 sequences. The number of anticodons for each amino acid range from 1 to 6 for different tRNAs. There was no correlation between number of anticodons per amino acid and number of tRF5.

Table 2 shows the number of genes in the bovine genome for each tRF. tRF5-His had the largest number per animal, but there are only 25 genes for the tRNA that produced it. Opposite to tRF5-His, tRF5-Gly had the largest number of genes in the genome $(n=1,795)$, and was the third most abundant. tRF5-Glu was the second most abundant, and it had 621 tRNA genes in the bovine genome. tRF5-Val and tRF5-Lys were the fourth and fifth in abundance, there were only 90 and 121 tRNA genes in the bovine genome.

Correlations between number of codons per amino acid, number of genes, and count of normalized tRF5s was determined. The correlation between number of codons and normalized tRF5s was $r=-0.12(P=0.59)$, and the correlation between number of genes and tRF5s was $0.39(P=0.08)$. Neither correlation was statistically significant; however, the latter correlation showed a tendency.

Table 3 shows the number of tRF3s. There were $562 \mathrm{tRF} 3 \mathrm{~s}$ in the animals under study. The number of tRF3s ranged from 41 to 107 , with an average of $70 \mathrm{tRF} 3 \mathrm{~s}$ per animal. The number of

TABLE 2 | Number of normalized 5' transfer RNA derived RNA fragments (tRF5) in each animal in the study.

\begin{tabular}{|c|c|c|c|c|c|c|c|c|c|c|c|}
\hline \multirow[b]{2}{*}{ tRF5 } & \multicolumn{8}{|c|}{ Animal ID } & \multirow{2}{*}{$\begin{array}{l}\text { Total } \\
\text { tRFs }\end{array}$} & \multirow{2}{*}{$\begin{array}{l}\text { tRFs } \\
\text { /cow }\end{array}$} & \multirow{2}{*}{$\begin{array}{l}\text { tRNA } \\
\text { genes }\end{array}$} \\
\hline & 1141 & 1143 & 2919 & 2927 & 2931 & 2938 & 2939 & 2942 & & & \\
\hline tRF5-Ala & 544 & 352 & 408 & 518 & 351 & 362 & 479 & 419 & 3433 & 429 & 83 \\
\hline tRF5-Arg & 2 & 0 & 1 & 0 & 0 & 1 & 0 & 0 & 4 & 0.5 & 204 \\
\hline tRF5-Asn & 0 & 0 & 0 & 0 & 0 & 0 & 1 & 0 & 1 & 0.125 & 40 \\
\hline tRF5-Asp & 12 & 5 & 18 & 4 & 9 & 8 & 6 & 2 & 64 & 8 & 54 \\
\hline tRF5-Cys & 27 & 21 & 30 & 94 & 9 & 43 & 27 & 25 & 276 & 35 & 510 \\
\hline tRF5-GIn & 152 & 432 & 313 & 359 & 394 & 307 & 455 & 307 & 2719 & 340 & 60 \\
\hline tRF5-Glu & 93648 & 88823 & 108094 & 78000 & 106609 & 78579 & 66925 & 71478 & 692156 & 86520 & 621 \\
\hline tRF5-Gly & 41814 & 56821 & 61251 & 60975 & 45043 & 60536 & 46838 & 49574 & 422852 & 52857 & 1795 \\
\hline tRF5-His & 75542 & 133863 & 116715 & 73717 & 140707 & 96272 & 111728 & 96764 & 845308 & 105664 & 25 \\
\hline tRF5-Ile & 0 & 0 & 0 & 0 & 0 & 0 & 0 & 0 & 0 & 0 & 25 \\
\hline tRF5-Leu & 3 & 1 & 4 & 5 & 1 & 1 & 2 & 0 & 17 & 2 & 49 \\
\hline tRF5-Lys & 5635 & 8000 & 6634 & 5972 & 5206 & 6484 & 6237 & 4596 & 48764 & 6096 & 121 \\
\hline tRF5-Met & 5 & 8 & 11 & 14 & 7 & 6 & 8 & 4 & 63 & 8 & 33 \\
\hline tRF5-Phe & 1 & 0 & 0 & 0 & 0 & 0 & 0 & 0 & 1 & 0.125 & 35 \\
\hline tRF5-Pro & 512 & 505 & 554 & 646 & 781 & 666 & 1277 & 1225 & 6166 & 771 & 26 \\
\hline tRF5-Ser & 0 & 0 & 1 & 1 & 0 & 0 & 0 & 0 & 2 & 0.25 & 79 \\
\hline tRF5-Stop & 94 & 25 & 94 & 28 & 35 & 71 & 51 & 38 & 436 & 55 & 25 \\
\hline tRF5-Thr & 24 & 13 & 14 & 8 & 11 & 15 & 18 & 8 & 111 & 14 & 132 \\
\hline tRF5-Trp & 0 & 0 & 0 & 0 & 0 & 0 & 0 & 0 & 0 & 0 & 182 \\
\hline tRF5-Tyr & 0 & 0 & 0 & 0 & 0 & 0 & 0 & 0 & 0 & 0 & 48 \\
\hline tRF5-Val & 34225 & 37307 & 44317 & 40587 & 31800 & 40946 & 54467 & 36366 & 320015 & 40002 & 90 \\
\hline Total tRF5 & 252240 & 326176 & 338459 & 260928 & 330963 & 284297 & 288519 & 260806 & 2342388 & 292799 & \\
\hline
\end{tabular}


TABLE 3 | Number of $3^{\prime}$ transfer RNA derived RNA Fragments (tRF3) in each animal in the study.

\begin{tabular}{|c|c|c|c|c|c|c|c|c|c|c|}
\hline \multirow[b]{2}{*}{ tRF3 } & \multicolumn{8}{|c|}{ Animal ID } & \multirow{2}{*}{$\begin{array}{l}\text { Total } \\
\text { tRFs }\end{array}$} & \multirow{2}{*}{$\begin{array}{l}\text { tRFs } \\
\text { /cow }\end{array}$} \\
\hline & 1141 & 1143 & 2919 & 2927 & 2931 & 2938 & 2939 & 2942 & & \\
\hline tRF3-Ala & 1 & 0 & 1 & 2 & 1 & 0 & 0 & 0 & 5 & 0.625 \\
\hline tRF3-Arg & 1 & 4 & 4 & 10 & 1 & 7 & 2 & 4 & 33 & 4.125 \\
\hline tRF3-Asn & 13 & 6 & 18 & 8 & 3 & 6 & 14 & 6 & 74 & 9.250 \\
\hline tRF3-Asp & 6 & 3 & 9 & 6 & 3 & 3 & 6 & 4 & 40 & 5 \\
\hline tRF3-Cys & 0 & 1 & 0 & 3 & 0 & 0 & 0 & 0 & 4 & 0.500 \\
\hline tRF3-Gln & 2 & 1 & 1 & 2 & 1 & 1 & 0 & 1 & 9 & 1.125 \\
\hline tRF3-Glu & 34 & 16 & 41 & 43 & 25 & 26 & 24 & 46 & 255 & 31.875 \\
\hline tRF3-Gly & 0 & 0 & 1 & 4 & 0 & 1 & 2 & 0 & 8 & 1 \\
\hline tRF3-His & 0 & 0 & 0 & 0 & 0 & 0 & 0 & 0 & 0 & 0 \\
\hline tRF3-Ile & 0 & 1 & 0 & 0 & 0 & 0 & 0 & 0 & 1 & 0.125 \\
\hline tRF3-Leu & 1 & 0 & 0 & 0 & 1 & 0 & 2 & 2 & 6 & 0.750 \\
\hline tRF3-Lys & 4 & 2 & 7 & 2 & 0 & 5 & 4 & 12 & 36 & 4.500 \\
\hline tRF3-Met & 3 & 2 & 1 & 1 & 1 & 1 & 0 & 0 & 9 & 1.125 \\
\hline tRF3-Phe & 0 & 1 & 0 & 0 & 0 & 0 & 1 & 0 & 2 & 0.25 \\
\hline tRF3-Pro & 0 & 1 & 1 & 3 & 0 & 0 & 0 & 0 & 5 & 0.625 \\
\hline tRF3-Ser & 0 & 0 & 0 & 0 & 0 & 0 & 0 & 0 & 0 & 0 \\
\hline tRF3-Stop & 0 & 0 & 0 & 0 & 0 & 0 & 0 & 0 & 0 & 0 \\
\hline tRF3-Thr & 1 & 0 & 3 & 7 & 1 & 3 & 2 & 1 & 18 & 2.250 \\
\hline tRF3-Trp & 2 & 0 & 1 & 0 & 1 & 0 & 1 & 0 & 5 & 0.625 \\
\hline tRF3-Tyr & 0 & 1 & 2 & 0 & 0 & 1 & 1 & 0 & 5 & 0.625 \\
\hline tRF3-Val & 6 & 2 & 5 & 16 & 3 & 7 & 2 & 6 & 47 & 5.875 \\
\hline Total tRF3 & 47 & 41 & 95 & 107 & 41 & 61 & 61 & 82 & 562 & \\
\hline
\end{tabular}

sequences was fewer than 100 in all cases. No sequences were observed for tRF3-His, tRF3-Ser, and tRF3-Stop. There were only three tRF1s identified in the study. Of the 81 sequences, 2 were tRF1-His, 76 were mitochondrial tRF1-Val, and 3 were mitochondrial tRF1-Tyr.

\section{Discussion}

It has been hypothesized that tRFs may be a significant regulator of gene silencing when animals are faced with a pathogen that may modify their homeostatic status, causing disease (Wang et al., 2013). A limited number of studies have evaluated tRFs in cell lines, mice, and plants (Cole et al., 2009; Loss-Morais et al., 2013; Maute et al., 2013; Kumar et al., 2014), and only one study has evaluated tRFs response to a bovine virus in bovine cell culture (Wang et al., 2013). The present study provides an overall characterization of tRFs that are circulating in cattle. This information is necessary to understand difference in expression profiles when pathogen challenge studies in live animals are conducted.

Production of tRFs has been observed when the cell is under stress (Zhang et al., 2014). The stress can be physical, chemical, or by a viral infection (Thompson and Parker, 2009; Wang et al., 2013). Zhang et al. (2014), using mice as an animal model, observed that during an acute inflammation process, there was an increase of serum tRFs during the first days of the inflammation, followed by a decrease a few days afterward. An initial screening of human patients with an active Hepatitis B virus infection also revealed an increase in serum tRFs (Zhang et al., 2014). Zhang et al. (2014) indicate that the biological significance and diagnostic value of serum tRFs warrants further studies and possibly opens a new round of research focused on serum tRFs. The objective of the present study was to characterize circulating tRFs in non-stressed bovine, with the ultimate goal to establish differences in tRF expression associated with diseases.

The tRFs have been classified according to their cleavage portion of tRNA present (Lee et al., 2009). Their production and role within the cell has been studied, but no indication of their relative abundance has been established in humans, animals, or plants (Garcia-Silva et al., 2012; Loss-Morais et al., 2013; Sobala and Hutvagner, 2013; Kumar et al., 2014). In the present study we have determined that under normal conditions, tRF5s are predominant, while tRF3 and tRF1 are present at a very low level in cattle sera. Wang et al. (2013), indicated that high throughput sequencing data captured tRF5, tRF3, and tRF1 in comparable quantities in prostate cancer cells. Increased production of tRF3 and tRF1 could be signs of changes in the homeostatic condition (i.e., disease), although further studies would be needed to establish this relationship.

The largest proportion of tRF5s was between 30 and 34 nucleotides in length, with tRF5s of 31 and 34 nucleotides being the most prevalent. Previous studies indicated that length of tRF5s was between 18 and 25 nucleotides (Lee et al., 2009) or between 21 and 22 nucleotides (Haussecker et al., 2010). Wang et al. (2013), identified a 31 nucleotide tRF5 (tRF5-Glu) as being a suppressor in messenger RNA in cytoplasm in the presence of bovine respiratory syncytial virus. It is unknown if differences in 
length are due to the location of the tRF5 (intra- or extra-cellular), if there are due to species difference in processing site, or are due to their function.

For each tRF5, the most abundant is almost double the amount of the next most abundant. As example, the most abundant tRF5his was 34 nucleotides $(n=553,427)$, while the second most abundant was present at $n=119,840$ (Table 1). This longest tRF5-his was produced 4.6 times greater than the second most common at 31 nucleotides. It will be necessary to further evaluate the function of each size of tRF5s to establish a comprehensive understanding of their function.

The association of number of tRNA genes in the genome, with tRF5s suggests that function rather than number is involved in production of tRFs. The most abundant tRF5s have a varying number of tRNA genes in the genome. The correlation between abundance of tRF5s and number of tRNA genes suggest that factors other than number of tRNA genes are influencing the production of tRF5s.

The sequence of each tRF is associated with a specific amino acid codon from which it originated. Function, rather than number of codons, should be associated with production of tRF5s. The tRF5-Glu identified by Wang et al. (2013) as being responsible for repressing mRNA in the cytoplasm is one of the most common in the present study. This is an indication that function drives the production of tRF5s. If the number of codons was to be responsible for production of tRF5s, amino acids with six codons should produce the largest amounts of tRF5s (tRF5-Arg, tRF5-Leu, and tRF5-Ser), which was not observed in the present study. Histidine, glutamine, glutamic acid, and lysine have two codons each, and the largest amounts of tRF5s were observed for their tRNAs. However, other amino acids with one or two codons (aspartic acid, aspargine, cysteine, phenylalanine, tryptophan, and tyrosine), had limited amounts of tRF5s. From amino acids with four codons, glycine and valine were the amino acids with the most tRF5s. However, other amino acids with four codons produced moderate numbers of tRF5s. The correlation between number of codons and amount of tRF5s produced by each amino acid is inconclusive. The reason why amino acids with the least number of codons have the most amounts of tRF5s can only be explained if these molecules are associated with function within the organism.

There is no association in production of tRF5s, tRF3s, and tRF1s. When Lee et al. (2009) described tRFs, they were able to clone and sequence equivalent number of sequences from three tRF families. Similarly, Sobala and Hutvagner (2013)

\section{References}

Bartel, D. P. (2009). MicroRNAs: target recognition and regulatory functions. Cell 136, 215-233. doi: 10.1016/j.cell.2009.01.002

Cole, C., Sobala, A., Lu, C., Thatcher, S. R., Bowman, A., Brown, J. W., et al. (2009). Filtering of deep sequencing data reveals the existence of abundant Dicer-dependent small RNAs derived from tRNAs. RNA 15, 2147-2160. doi: 10.1261/rna.1738409

de Sá, P. H., Veras, A. A., Carneiro, A. R., Pinheiro, K. C., Pinto, A. C., Soares, S. C., et al. (2015). The impact of quality filter for RNA-Seq. Gene 563, 165-171. doi: $10.1016 /$ j.gene.2015.03.033 and Kumar et al. (2014) observed similar proportions of the three tRFs families. The previously mentioned studies were done from cells grown in vitro. Production of tRFs in live organisms may be different, evidenced by the results of the present study and may not necessarily reflect what takes place in the animal.

There were several tRF5s with few sequences. The depth of coverage was adequate for the needs of the study according to Haas et al. (2012). Sequencing error rates should have occurred in the present study (Wang et al., 2012); however, there was no attempt to eliminate those tRFs with few sequences (de Sá et al., 2015). tRFs with low number of sequences could potentially be spurious results and should be taken into account when interpreting results from this study.

The largest number of sequences observed in the present study ranged from 29 to 35 nucleotides in length. Sequences ranging from 15 to 27 nucleotides in length were removed from the study due to their low frequency. Kumar et al. (2014) reported tRFs ranging from 14 to 32 nucleotides in length. Kumar et al. (2014) analyzed sequences from the NCBI SRA database and mapping them to the human, mouse, Drosophila, Caenorhabditis elegans, Saccharomyces cerevisiae, and Schizosaccharomyces pombe, while the present study comprised cattle sequences. It is possible that length of tRF5s is associated with the species.

Identification of tRFs is a relatively new field which is expanding due to the availability of high throughput sequencing. This is also the case for livestock production and health. We are embarking in identification of small non-coding RNA studies to establish their association with animals affected with diseases. The results from the present study could potentially be used as reference in cattle.

\section{Author Contributions}

EC, GC, and JN conceived the project and interpreted results. EC and GC performed the experiment. EC wrote, and GC and JN reviewed the manuscript.

\section{Acknowledgments}

The authors thank Randy Atchison from the NADC, for excellent technical assistance, and Dr. John Lippolis for providing the blood samples.

Garcia-Silva, M. R., Cabrera-Cabrera, F., Guida, M. C., and Cayota, A. (2012). Hints of tRNA-derived small RNAs role in RNA silencing mechanisms. Genes (Basel) 3, 603-614. doi: 10.3390/genes3040603

Haas, B. J., Chin, M., Nusbaum, C., Birren, B. W., and Livny, J. (2012). How deep is deep enough for RNA-Seq profiling of bacterial transcriptomes? BMC Genomics 13:734. doi: 10.1186/1471-2164-13-734

Haussecker, D., Huang, Y., Lau, A., Parameswaran, P., Fire, A. Z., and Kay, M. A. (2010). Human trna-derived small RNAs in the global regulation of RNA silencing. RNA 16, 673-695. doi: 10.1261/rna.2000810

Ivanov, P., Emara, M. M., Villen, J., Gygi, S. P., and Anderson, P. (2011). Angiogenin-induced tRNA fragments inhibit translation 
initiation. Mol. Cell 43, 613-623. doi: 10.1016/j.molcel.2011. 06.022

Kumar, P., Anaya, J., Mudunuri, S. B., and Dutta, A. (2014). Meta-analysis of trna derived RNA fragments reveals that they are evolutionarily conserved and associate with AGO proteins to recognize specific RNA targets. BMC Biol. 12:78. doi: 10.1186/s12915-014-0078-0

Lee, Y. S., Shibata, Y., Malhotra, A., and Dutta, A. (2009). A novel class of small RNAs: tRNA-derived RNA fragments (tRFs). Genes Dev. 23, 2639-2649. doi: 10.1101/gad.1837609

Loss-Morais, G., Waterhouse, P. M., and Margis, R. (2013). Description of plant tRNA-derived RNA fragments (tRFs) associated with argonaute and identification of their putative targets. Biol. Dir. 8:6. doi: 10.1186/17456150-8-6

Maute, R. L., Schneider, C., Sumazin, P., Holmes, A., Califano, A., Basso, K., et al. (2013). tRNA-derived microRNA modulates proliferation and the DNA damage response and is down-regulated in B cell lymphoma. Proc. Natl. Acad. Sci. U.S.A. 110, 1404-1409. doi: 10.1073/pnas.12067 61110

Nolte-'t Hoen, E. N., Buermans, H. P., Waasdorp, M., Stoorvogel, W., Wauben, M. H., and Hoen, P. A. (2012). Deep sequencing of RNA from immune cell-derived vesicles uncovers the selective incorporation of small non-coding RNA biotypes with potential regulatory functions. Nucleic Acids Res. 40, 9272-9285. doi: 10.1093/nar/ gks658

Sobala, A., and Hutvagner, G. (2013). Small RNAs derived from the 5 ' end of tRNA can inhibit protein translation in human cells. RNA Biol. 10, 553-563. doi: $10.4161 /$ rna. 24285
Stewart, C. R., Marsh, G. A., Jenkins, K. A., Gantier, M. P., Tizard, M. L., Middleton, D., et al. (2013). Promotion of Hendra virus replication by microRNA 146a. J. Virol. 87, 3782-3791. doi: 10.1128/JVI. 01342-12

Thompson, D. M., and Parker, R. (2009). Stressing out over tRNA cleavage. Cell 138, 215-219. doi: 10.1016/j.cell.2009.07.001

Wang, Q., Lee, I., Ren, J., Ajay, S. S., Lee, Y. S., and Bao, X. (2013). Identification and functional characterization of tRNA-derived RNA fragments (tRFs) in respiratory syncytial virus infection. Mol. Ther. 21, 368-379. doi: $10.1038 / \mathrm{mt} .2012 .237$

Wang, X. V., Blades, N., Ding, J., Sultana, R., and Parmigiani, G. (2012). Estimation of sequencing error rates in short reads. BMC Bioinform. 13:185. doi: 10.1186/1471-2105-13-185

Zhang, Y., Zhang, Y., Shi, J., Zhang, H., Cao, Z., Gao, X., et al. (2014). Identification and characterization of an ancient class of small RNAs enriched in serum associating with active infection. J. Mol. Cell Biol. 6, 172-174. doi: $10.1093 / \mathrm{jmcb} / \mathrm{mjt} 052$

Conflict of Interest Statement: Eduardo Casas, Guohong Cai, and John D. Neill are USDA employees and declare no conflict of interest.

Copyright (c) 2015 Casas, Cai and Neill. This is an open-access article distributed under the terms of the Creative Commons Attribution License (CC BY). The use, distribution or reproduction in other forums is permitted, provided the original author(s) or licensor are credited and that the original publication in this journal is cited, in accordance with accepted academic practice. No use, distribution or reproduction is permitted which does not comply with these terms. 